\title{
PERTURBATION OF THE GENERALIZED DRAZIN INVERSE*
}

\author{
CHUNYUAN DENG ${ }^{\dagger}$ AND YIMIN WEI ${ }^{\ddagger}$
}

\begin{abstract}
In this paper, we investigate the perturbation of the generalized Drazin invertible matrices and derive explicit generalized Drazin inverse expressions for the perturbations under certain restrictions on the perturbing matrices.
\end{abstract}

Key words. generalized Drazin inverse, perturbation, index, block matrix.

AMS subject classifications. 47A05, 15A09.

1. Introduction. The Drazin inverse of an operator or a matrix has various applications in singular differential equations and singular difference equations, Markov chains, and iterative methods (see $[1,2,3,4,16,17,22,23,30]$ ). In addition, the perturbation analysis of the Drazin inverse is important from the perspectives of both pure and computational mathematics (see [2]-[4],[6]-[7],[19]-[29]). The perturbation results of the Drazin inverse of a closed linear operator can be applied to the solution of perturbed linear equations and to the asymptotic behavior of $\mathcal{C}_{0}$-semigroup of bounded linear operators $[2,16]$.

Let $X$ denote an arbitrary complex Banach space and $\mathcal{B}(X)$ denote the Banach algebra of all linear bounded operators on $X$. If $A \in \mathcal{B}(X)$, then $\mathcal{R}(A), \mathcal{N}(A), \sigma(A)$ and $\gamma(A)$ denote the range, the kernel, the spectrum and the spectral radius of $A$, respectively. Recall that an element $B \in \mathcal{B}(X)$ is the Drazin inverse of $A \in \mathcal{B}(X)$ provided that

$$
A^{k+1} B=A^{k}, \quad B A B=B \quad \text { and } \quad A B=B A
$$

hold for some nonnegative integer $k$. The smallest $k$ in the definition is called the Drazin index of $A$, and is denoted by $i(A)$. If $A$ has the Drazin inverse, then the Drazin

${ }^{*}$ Received by the editors on June 10, 2009. Accepted for publication on July 31, 2010. Handling Editors: Roger A. Horn and Fuzhen Zhang.

${ }^{\dagger}$ School of Mathematics Science, South China Normal University, Guangzhou 510631, P. R. China (cydeng@scnu.edu.cn). Supported by a grant from the Ph.D. Programs Foundation of Ministry of Education of China (No. 20094407120001 ).

${ }^{\ddagger}$ Corresponding author: Y. Wei (ymwei@fudan.edu.cn and yimin.wei@gmail.com). School of Mathematical Sciences and Shanghai Key Laboratory of Contemporary Applied Mathematics, Fudan University, Shanghai, 200433, P. R. China. Supported by the National Natural Science Foundation of China under grant 10871051, Shanghai Municipal Science \& Technology Committee under grant 09DZ2272900, Shanghai Municipal Education Committee (Dawn Project), Doctoral Program of the Ministry of Education under grant 20090071110003 and KLMM0901. 
inverse of $A$ is unique and is denoted by $A^{D}$. It is well-known that $A \in \mathcal{B}(X)$ has the Drazin inverse if and only if the point $\lambda=0$ is a pole of the resolvent $\lambda \mapsto(\lambda I-A)^{-1}$. The order of this pole is equal to $i(A)$. Particularly, it follows that 0 is not the accumulation point of the spectrum $\sigma(A)$ (see $[1,13,17,18]$ ). For a compact subset $\mathcal{M}$ of $\mathbb{C}$, we use acc $\sigma(\mathcal{M})$ to denote the set of all points of accumulation of $\mathcal{M}$.

In [18], Koliha introduced the concept of a generalized Drazin inverse (or GDinverse). The GD-inverse of an element $A \in \mathcal{B}(X)$ exists if and only if $0 \notin$ acc $\sigma(A)$. If $0 \notin$ acc $\sigma(A)$, then there exist open subsets $U$ and $V$ of $\mathbb{C}$, such that $\sigma(A) \backslash\{0\} \subset U$, $0 \in V$ and $U \cap V=\emptyset$. Define a function $f$ in the following way:

$$
f(\lambda)= \begin{cases}0, & \lambda \in V \\ \frac{1}{\lambda}, & \lambda \in U .\end{cases}
$$

The function $f$ is regular in a neighborhood of $\sigma(A)$, i.e., it is analytic and singlevalued throughout a neighborhood of $\sigma(A)$. The GD-inverse of $A$ is defined as $A^{d}=$ $f(A)$. If $A$ is GD-invertible, then the spectral idempotent $A^{\pi}$ of $A$ corresponding to $\{0\}$ is defined by $A^{\pi}=I-A A^{d}$. The matrix form of $A$ with respect to the decomposition $X=\mathcal{N}\left(A^{\pi}\right) \oplus \mathcal{R}\left(A^{\pi}\right)$ is given by

$$
A=A_{1} \oplus A_{2},
$$

where $A_{1}$ is invertible and $A_{2}$ is quasinilpotent. If we denote $C_{A}=A_{1} \oplus O$ and $Q_{A}=O \oplus A_{2}$, then $A=C_{A}+Q_{A}$ is known as the core-quasinilpotent decomposition of $A$.

$$
U=\left(\begin{array}{cc}
U_{11} & U_{12} \\
O & U_{22}
\end{array}\right) \quad \text { and } \quad B=A+U=\left(\begin{array}{cc}
A_{1}+U_{11} & U_{12} \\
O & A_{2}+U_{22}
\end{array}\right)
$$

with respect to the decomposition $X=\mathcal{N}\left(A^{\pi}\right) \oplus \mathcal{R}\left(A^{\pi}\right)$. In our paper, we consider the perturbation of the generalized Drazin inverses in more generalized cases as follows:

- $A_{1}+U_{11}$ is invertible and $\operatorname{dim}\left[\mathcal{R}\left(A^{\pi}\right)\right]$ is finite.

- $A_{1}+U_{11}$ is invertible and $U_{22} A_{2}=O$.

- $A_{1}+U_{11}$ is invertible and $U_{22} A_{2}=A_{2} U_{22}$.

In the existing literature $[2,12,19,24,25,26,27,28,29]$, only are the following cases considered:

- $\left\|I+A_{1}^{-1} U_{11}\right\|<1$, which ensures that $A_{1}+U_{11}$ is invertible, $A_{2}+U_{22}$ is quasinilpotent and $U_{12}=O$. 
- $A_{1}+U_{11}$ is invertible, $U_{22}$ is quasinilpotent with $A_{2} U_{22}=U_{22} A_{2}$ and $U_{12}=O$.

- $U_{11}=O, U_{22}$ is quasinilpotent and $U_{22} A_{2}=O$.

- $\left\|I+A_{1}^{-1} U_{11}\right\|<1, U_{12}=O$ and $U_{22}=O$.

The main purpose of our paper is to study the perturbation of the GD-inverse $A^{d}$ of a bounded linear operator $A$ and to obtain a bound for $\| A+U)^{d}-A^{d} \|$ under certain conditions on the perturbing operator $U$, usually of small perturbation. The error bounds for the perturbed GD-inverse without restriction on the perturbing matrix (or operator) are difficult to obtain. We derive explicit expressions of GD-inverse for the perturbations under certain restrictions on the perturbing operators.

2. Main results and proofs. To prove the main results, we begin with some lemmas.

Lemma 2.1. [15, Corollary 8] Let $\left(\begin{array}{ll}A & B \\ O & C\end{array}\right)$ be a bounded linear operator on $X \oplus Y$. If $\sigma(A) \cap \sigma(C)$ has no-interior point, then

$$
\sigma\left[\left(\begin{array}{cc}
A & B \\
O & C
\end{array}\right)\right]=\sigma(A) \cup \sigma(C)
$$

For $\mu \in \mathbb{C}$ and $K \subset \mathbb{C}$, we define $\operatorname{dist}(\mu, K)=\inf \{|\lambda-\mu|: \lambda \in K\}$ if $K \neq \emptyset$ and $\operatorname{dist}(\mu, \emptyset)=\infty$. The following result establishes the spectral characterization of perturbation operators.

Lemma 2.2. If $A$ and $U \in \mathcal{B}(X), B=A+U$ and $\sigma_{\epsilon}(A)=\{\lambda: \operatorname{dist}(\lambda, \sigma(A))<\epsilon\}$, then for every $\epsilon>0$ there exists $\delta>0$ such that $\sigma(B) \subset \sigma_{\epsilon}(A)$ whenever $\|U\|<\delta$.

Proof. First we can suppose that $\delta=1$. Then it is clear that $\sigma(A)$ and $\sigma(B)$ are in the closed disk $D=\{\lambda:|\lambda| \leq 1+\|B\|\}$. For every $\epsilon, D \backslash \sigma_{\epsilon}(A)$ is a closed subset in complex plane. If $\mu \in D \backslash \sigma_{\epsilon}(A)$, then $\mu I-A$ is invertible. So there exists an open ball $\mathbb{U}\left(\mu I-A, \delta_{\mu}\right) \subset \mathcal{B}(X)$ such that $T$ is invertible for all $T \in \mathbb{U}\left(\mu I-A, \delta_{\mu}\right)$.

Let $E_{\mu}=\left\{\lambda:|\lambda-\mu|<\frac{1}{2} \delta_{\mu}\right\}$. Then $\left\{E_{\mu}: \mu \in D \backslash \sigma_{\epsilon}(A)\right\}$ is an open covering of the compact subset $D \backslash \sigma_{\epsilon}(A)$. So there is a finite subcovering $\left\{E_{\mu_{i}}, i=1,2, \ldots, n\right\}$ covering the subset $D \backslash \sigma_{\epsilon}(A)$. Put $\delta=\min \left\{1, \frac{1}{2} \delta_{\mu_{i}}, i=1,2, \ldots, n\right\}$. For every $\lambda \in$ $D \backslash \sigma_{\epsilon}(A)$, if $\|U\|<\delta$, then there exists an open subset $E_{\mu_{i}}$ so that $\lambda \in E_{\mu_{i}}$. From $\left\|\lambda I-B-\left(\mu_{i} I-A\right)\right\| \leq\|A-B\|+\left|\lambda-\mu_{i}\right|<\delta+\frac{1}{2} \delta_{\mu_{i}}<\delta_{\mu_{i}}$, we have $\lambda I-B \in$ $\mathbb{U}\left(\mu_{i} I-A, \delta_{\mu_{i}}\right)$. It follows that $\lambda \notin \sigma(B)$ and therefore $\sigma(B) \subset \sigma_{\epsilon}(A)$. $\square$

If $A \in \mathcal{B}(X)$ and $\gamma(A)>0$, Koliha and Rakočević got the following basic lemma $[17$, Lemma 1.3].

Lemma 2.3. [17, Lemma 1.3] Let $A \in \mathcal{B}(X)$ be Drazin invertible. If $\gamma(A)>0$, 
then

$$
\operatorname{dist}(0, \sigma(A) \backslash\{0\})=\left(\gamma\left(A^{D}\right)\right)^{-1}
$$

Additive perturbation results for the GD-inverse were investigated by many authors (see $[5,7,8,12,13,16])$. In [12], a detailed treatment of the generalized Drazin inverse $(A+B)^{d}$ in terms of $A^{d}$ and $B^{d}$ in infinite dimensional spaces was given.

LEMma 2.4. (1) [12, Theorem 2.3] Let $A$ and $B \in \mathcal{B}(X)$ be GD-invertible with $A B=O$. Then $A+B$ is GD-invertible and

$$
(A+B)^{d}=\left(I-B B^{d}\right)\left[\sum_{n=0}^{\infty} B^{n}\left(A^{d}\right)^{n}\right] A^{d}+B^{d}\left[\sum_{n=0}^{\infty}\left(B^{d}\right)^{n} A^{n}\right]\left(I-A A^{d}\right) .
$$

(2) $[12$, Theorem 2.1] Let $A, B, A+B \in \mathcal{B}(X)$ be $G D$-invertible and $A B=B A$. Then $A+B$ is GD-invertible and

$$
(A+B)^{d}=\left(C_{A}+C_{B}\right)^{d}\left[I+\left(C_{A}+C_{B}\right)^{d}\left(Q_{A}+Q_{B}\right)\right]^{-1},
$$

where $A=C_{A}+Q_{A}$ and $B=C_{B}+Q_{B}$ are known as the core-quasinilpotent decomposition of $A$ and $B$, respectively.

Lemma 2.5. Let $X$ be infinite dimensional Banach space. If $A \in \mathcal{B}(X)$ is a nilpotent operator, then $\operatorname{dim}[\mathcal{N}(A)]=\infty$.

Proof. If $A=O$, it is obvious that $\operatorname{dim}[\mathcal{N}(A)]=\infty$. If $A$ is non-zero nilpotent, then there exists a positive integer $k \geq 2$ such that $A^{k}=O$ and $A^{k-1} \neq O$. Suppose to the contrary that $\operatorname{dim}[\mathcal{N}(A)]=n_{0}<\infty$, then $\operatorname{dim}[\mathcal{R}(A)]=\infty$. It is clear that $\operatorname{dim}[\mathcal{N}(A) \cap \mathcal{R}(A)] \leq n_{0}$, which shows that $\operatorname{dim}\left[\mathcal{R}\left(A^{2}\right)\right]=\infty$. By induction, it is easy to see that $\operatorname{dim}\left[\mathcal{R}\left(A^{k-1}\right)\right]=\infty$ and $\mathcal{R}\left(A^{k-1}\right) \subseteq \mathcal{N}(A)$. This is a contradiction.

For the Drazin index, if $A$ and $B$ are quasinilpotent, then $i(A)$ and $i(B) \in \mathbb{N} \cup \infty$. The following results were first obtained by González and Koliha for the case that $A$ and $B$ are commuting quasinilpotent elements (see [2]). In fact, if both $i(A)$ and $i(B)$ are finite or exactly one of $i(A)$ and $i(B)$ is finite $(\infty-\infty$ is not defined), then we have the following generalization.

LEMma 2.6. Let $A, B$ be quasinilpotent. If $A B \in\left\{O, \pm A^{2}, \pm B^{2}, \pm B A\right\}$, then

$$
|i(A)-i(B)|+1 \leq i(A+B) \leq i(A)+i(B)-1
$$

holds provided $i(A)>0$ and the difference $i(A)-i(B)$ is defined.

Proof. It is easy to obtain

$$
(A+B)^{m}=\sum_{i=0}^{m} B^{i} A^{m-i} \text { if } A B=O
$$


and

$$
(A+B)^{m}=\frac{1}{2} B^{m}+\sum_{i=0}^{m} 2^{m-i-1} B^{i} A^{m-i} \text { if } A B=A^{2},
$$

we have $i(A+B) \leq i(A)+i(B)-1$. Similarly we have $i(A+B) \leq i(A)+i(B)-1$ if $A B \in\left\{-A^{2}, \pm B^{2}, \pm B A\right\}$.

If $A B \in\left\{O, \pm A^{2}, \pm B^{2}, \pm B A\right\}$, in a similar way to the proof of the first step, we have $i(A)=i((A+B)+(-B)) \leq i(A+B)+i(B)-1, i(B)=i((-A)+(A+B)) \leq$ $i(A+B)+i(B)-1$. So we obtain $|i(A)-i(B)|+1 \leq i(A+B)$ whenever the difference $i(A)-i(B)$ is defined.

The following result has been proved in [12].

Lemma 2.7. [12] If $A \in \mathcal{B}(X)$ and $B \in \mathcal{B}(Y)$ are $G D$-invertible and $C \in \mathcal{B}(Y, X)$, then $M=\left(\begin{array}{cc}A & C \\ O & B\end{array}\right)$ is $G D$-invertible and $M^{d}=\left(\begin{array}{cc}A^{d} & X \\ O & B^{d}\end{array}\right)$, where

$$
X=\left[\sum_{n=0}^{\infty}\left(A^{d}\right)^{n+2} C B^{n}\right] B^{\pi}+A^{\pi}\left[\sum_{n=0}^{\infty} A^{n} C\left(B^{d}\right)^{n+2}\right]-A^{d} C B^{d} .
$$

First we discuss the GD-invertibility of the finite rank perturbation.

Theorem 2.8. Let $A \in \mathcal{B}(X)$ be $G D$-invertible, $U \in \mathcal{B}(X)$ satisfy $A^{\pi} U\left(I-A^{\pi}\right)=$ $O$. There exists a $\delta>0$ such that $B=A+U$ is Drazin invertible for each $\|U\|<\delta$ if and only if $\operatorname{dim}\left[\mathcal{R}\left(A^{\pi}\right)\right]$ is finite.

Proof. "Necessity." Let $P=A^{\pi}$ be the spectral idempotent of $A$ corresponding to $\{0\}$. Then $A$ has the matrix form

$$
A=A_{1} \oplus A_{2}
$$

with respect to the decomposition $X=\mathcal{N}(P) \oplus \mathcal{R}(P)$, where $A_{1}$ is invertible and $A_{2}$ is quasinilpotent. If $\operatorname{dim}\left[\mathcal{R}\left(A^{\pi}\right)\right]$ is infinite, by Lemma $2.5, \operatorname{dim}\left[\mathcal{N}\left(A_{2}\right)\right]=\infty$. Then $A_{2}$ has the following operator matrix form

$$
A_{2}=\left(\begin{array}{cc}
O & A_{23} \\
O & A_{33}
\end{array}\right)
$$

with respect to the space decomposition $\mathcal{R}(P)=\mathcal{N}\left(A_{2}\right) \oplus\left(\mathcal{R}(P) \ominus \mathcal{N}\left(A_{2}\right)\right)$, where $A_{33}$ is quasinilpotent. If $S$ is a unilateral shift defined on $\mathcal{N}\left(A_{2}\right)$, then $\sigma(S)$ is a unit disk. Define $U$ by $U=O \oplus \frac{1}{2 \delta} S \oplus O$. Then $\|U\|<\delta$ and

$$
A+U=\left(\begin{array}{ccc}
A_{1} & O & O \\
O & \frac{1}{2 \delta} S & A_{23} \\
O & O & A_{33}
\end{array}\right)
$$


with respect to the space decomposition $X=\mathcal{N}(P) \oplus \mathcal{N}\left(A_{2}\right) \oplus\left(\mathcal{R}(P) \ominus \mathcal{N}\left(A_{2}\right)\right)$. Thus $\sigma(A+U)=\sigma\left(A_{1}\right) \cup \sigma\left(\frac{1}{2 \delta} S\right)$ by Lemma 2.1. This shows that 0 is not an isolated point of $\sigma(A+U)$. Therefore $B=A+U$ is not Drazin invertible.

"Sufficiency." If $A$ is invertible, then the result holds. If $A$ is quasinilpotent, then $\operatorname{dim}(X)$ is finite because $\operatorname{dim}\left[\mathcal{R}\left(A^{\pi}\right)\right]$ is finite. Since all operators in $\mathcal{B}(X)$ are Drazin invertible when $\operatorname{dim}(X)$ is finite, the result is obvious.

Suppose that $A \in \mathcal{B}(X)$ is GD-invertible, neither invertible nor quasinilpotent. If $A$ has the operator matrix form (2.1), then $\sigma(A)=\sigma\left(A_{1}\right) \cup\{0\}$, and $\sigma\left(A_{1}\right) \cap\{0\}=\emptyset$, where $\sigma\left(A_{2}\right)=\{0\}$. By Lemma 2.3, we have

$$
\operatorname{dist}(0, \sigma(A) \backslash\{0\})=\left(\gamma\left(A^{d}\right)\right)^{-1}>0 .
$$

For small enough $\epsilon>0$, there exists two disjoint closed subsets $M_{1}$ and $M_{2}$ such that $\sigma_{\epsilon}\left(A_{1}\right)=\left\{\lambda: \operatorname{dist}\left(\lambda, \sigma\left(A_{1}\right)\right)<\epsilon\right\}$ and $\sigma_{\epsilon}\left(A_{2}\right)=\left\{\lambda: \operatorname{dist}\left(\lambda, \sigma\left(A_{2}\right)\right)<\epsilon\right\}$ are contained in the interior of $M_{1}$ and $M_{2}$, respectively.

Since $A^{\pi} U\left(I-A^{\pi}\right)=O, U$ and $B$ have the following operator matrix forms

$$
U=\left(\begin{array}{cc}
U_{11} & U_{12} \\
O & U_{22}
\end{array}\right), \quad B=\left(\begin{array}{cc}
A_{1}+U_{11} & U_{12} \\
O & A_{2}+U_{22}
\end{array}\right)
$$

with respect to the decomposition $X=\mathcal{N}\left(A^{\pi}\right) \oplus \mathcal{R}\left(A^{\pi}\right)$. By Lemma 2.2, there is a $\delta>0$ such that $\sigma\left(A_{1}+U_{11}\right) \subset \sigma_{\epsilon}\left(A_{1}\right) \subset M_{1}$ and $\sigma\left(A_{2}+U_{22}\right) \subset \sigma_{\epsilon}\left(A_{2}\right) \subset M_{2}$ whenever $\|U\|<\delta$. Thus $\sigma(B)=\sigma\left(A_{1}+U_{11}\right) \cup \sigma\left(A_{2}+U_{22}\right)$ by Lemma 2.1. Since $\operatorname{dim}\left[\mathcal{R}\left(A^{\pi}\right)\right]$ is finite, $\sigma\left(A_{2}+U_{22}\right)$ is a finite subset of $\sigma(B)$. Note that $0 \notin \sigma\left(A_{1}+U_{11}\right)$. So 0 is not an accumulated point of $\sigma(B)$. Therefore $B=A+U$ is Drazin invertible.

Definition 2.9. Let $A \in \mathcal{B}(X)$ be GD-invertible and $\delta>0$.

(i) An operator $U \in \mathcal{B}(X)$ is called $A_{\delta}$-compatible if it satisfies $A^{\pi} U\left(I-A^{\pi}\right)=O$ and if $A^{\pi} U$ is GD-invertible for each $\|U\|<\delta$.

(ii) If the operator $U$ is $A_{\delta}$-compatible and satisfies $A A^{\pi} U=O$, then we say $U$ is $A_{\delta}^{0}$-compatible.

(iii) If the operator $U$ is $A_{\delta}$-compatible and satisfies $A A^{\pi} U=A^{\pi} U A$, and -1 is not the accumulation point of $\sigma\left[\left(A^{\pi} U\right)^{d} A^{\pi} U\left(I+\left(A^{\pi} U\right)^{d} A\right)\right]$, then we say $U$ is $A_{\delta}^{-1}$-compatible.

Next we consider the perturbation of the generalized Drazin inverse. We need some notation. Let

$$
\nu_{A}=\left(I+A^{d} U\right)^{-1} A^{d},
$$




$$
\begin{aligned}
& \kappa_{A}=C_{A^{\pi} U}^{d}\left(I+C_{A^{\pi} U}^{d} A\right)^{-1} \\
& \omega_{A}=\left(A^{\pi} U\right)^{d}\left(\sum_{n=0}^{\infty}\left(\left(A^{\pi} U\right)^{d}\right)^{n}\left(A^{\pi} A\right)^{n}\right) .
\end{aligned}
$$

Theorem 2.10. Let $A \in \mathcal{B}(X)$ be GD-invertible. Then there always exists a $\delta>0$ such that $A+U$ is $G D$-invertible whenever $U \in \mathcal{B}(X)$ is $A_{\delta}^{0}$-compatible. In this case, we have

$$
(A+U)^{d}=\nu_{A}+\omega_{A}-\nu_{A} U \omega_{A}+\left[\sum_{n=0}^{\infty} \nu_{A}^{n+2} U A^{\pi}\left(A^{\pi}(A+U)\right)^{n}\right] \times\left[I-(A+U) \omega_{A}\right]
$$

and the inequality

$$
\left|i(A)-i\left(A^{\pi} U\right)\right| \leq i(A+U) \leq i(A)+i\left(A^{\pi} U\right)-1
$$

holds provided $i(A)>0$ and the difference $i(A)-i\left(A^{\pi} U\right)$ is defined.

Proof. Let $B=A+U$ and $P=A^{\pi}$ be the spectral idempotent of $A$ corresponding to $\{0\}$. If $U$ is $A_{\delta}^{0}$-compatible, then $A, U$ and $B$ have the matrix forms

$$
A=\left(\begin{array}{cc}
A_{1} & O \\
O & A_{2}
\end{array}\right) ; \quad U=\left(\begin{array}{cc}
U_{11} & U_{12} \\
O & U_{22}
\end{array}\right) ; \quad B=\left(\begin{array}{cc}
A_{1}+U_{11} & U_{12} \\
O & A_{2}+U_{22}
\end{array}\right)
$$

with respect to the decomposition $X=\mathcal{N}(P) \oplus \mathcal{R}(P)$, where $A_{1}$ is invertible, $A_{2}$ is quasinilpotent, $U_{22}$ is GD-invertible and $A_{2} U_{22}=O$.

If $A$ is invertible, then $i(A)=0$ and $A^{\pi}=O$. By Lemma 2.1, there exists a $\delta>0$ such that $B$ is invertible, $B^{-1}=\left(I+A^{-1} U\right)^{-1} A^{-1}$ and $i(B)=0$. The results hold. Similarly, by Lemma 2.4 and Lemma 2.6, we can show that the results hold if $A$ is quasinilpotent.

Now suppose that $A \in \mathcal{B}(X)$ is GD-invertible with $i(A)>0$ and $\sigma(A) \neq\{0\}$. For small enough $\epsilon>0$, there are two disjoint closed subsets $M_{1}$ and $M_{2}$ such that $\sigma_{\epsilon}\left(A_{1}\right) \subset M_{1}$ and $\sigma_{\epsilon}\left(A_{2}\right) \subset M_{2}$. By Lemma 2.2, there exists a $\delta>0$ such that $\sigma\left(A_{1}+U_{11}\right) \subset \sigma_{\epsilon}\left(A_{1}\right)$ and $\sigma\left(A_{2}+U_{22}\right) \subset \sigma_{\epsilon}\left(A_{2}\right)$ whenever $\|U\|<\delta$ (this implies that $\left\|U_{11}\right\|<\delta$ and $\left.\left\|U_{22}\right\|<\delta\right)$. So we have $\sigma\left(A_{1}+U_{11}\right) \cap \sigma\left(A_{2}+U_{22}\right)=\emptyset$. It follows that $\sigma(B)=\sigma\left(A_{1}+U_{11}\right) \cup \sigma\left(A_{2}+U_{22}\right)$ by Lemma 2.1. Hence, there always exists a $\delta>0$ such that $A_{1}+U_{11}$ is invertible whenever $\|U\|<\delta$.

Since $A^{\pi} U$ is GD-invertible and $A A^{\pi} U=O, A_{2}+U_{22}$ is GD-invertible and

$$
\left(A_{2}+U_{22}\right)^{d}=U_{22}^{d}\left[\sum_{n=0}^{\infty}\left(U_{22}^{d}\right)^{n} A_{2}^{n}\right]
$$


by Lemma 2.4. Hence, there exists a $\delta>0$ such that $A_{1}+U_{11}$ is invertible and $A_{2}+U_{22}$ is GD-invertible whenever $\|U\|<\delta$. It follows that $B=A+U$ is GD-invertible. By Lemma 2.7, we have

$$
B^{d}=\left(\begin{array}{cc}
\left(A_{1}+U_{11}\right)^{-1} & S \\
O & \left(A_{2}+U_{22}\right)^{d}
\end{array}\right),
$$

where

$\left.S=\sum_{n=0}^{\infty}\left(A_{1}+U_{11}\right)^{-(n+2)} U_{12}\left(A_{2}+U_{22}\right)^{n}\right]\left(A_{2}+U_{22}\right)^{\pi}-\left(A_{1}+U_{11}\right)^{-1} U_{12}\left(A_{2}+U_{22}\right)^{d}$.

Note that $\left(A_{1}+U_{11}\right)^{-1} \oplus O=\left(I+A^{d} U\right)^{-1} A^{d}=\nu_{A}$,

$$
\begin{aligned}
{\left[A^{\pi}(A+U)\right]^{d} } & =O \oplus\left(A_{2}+U_{22}\right)^{d}=O \oplus U_{22}^{d}\left[\sum_{n=0}^{\infty}\left(U_{22}^{d}\right)^{n} A_{2}^{n}\right] \\
& =\left(A^{\pi} U\right)^{d}\left[\sum_{n=0}^{\infty}\left(\left(A^{\pi} U\right)^{d}\right)^{n}\left(A^{\pi} A\right)^{n}\right] \\
& =\omega_{A}
\end{aligned}
$$

and

$$
\left(\begin{array}{ll}
O & S \\
O & O
\end{array}\right)=\left[\sum_{n=0}^{\infty} \nu_{A}^{n+2} U A^{\pi}\left(A^{\pi}(A+U)\right)^{n}\right] \times\left[I-(A+U) \omega_{A}\right]-\nu_{A} U \omega_{A} .
$$

So we get

$$
B^{d}=\nu_{A}+\omega_{A}-\nu_{A} U \omega_{A}+\left[\sum_{n=0}^{\infty} \nu_{A}^{n+2} U A^{\pi}\left(A^{\pi}(A+U)\right)^{n}\right] \times\left[I-(A+U) \omega_{A}\right] .
$$

Note that $A=A_{1} \oplus A_{2}$ and $A_{1}+U_{11}$ is invertible. Then $i(A)=i\left(A_{2}\right)$ and $i(B)=i\left(A_{2}+U_{22}\right)$. Since $A_{2}$ is quasinilpotent, $U_{22}$ is GD-invertible and $A_{2} U_{22}=O$, we have

$$
A_{2}=\left(\begin{array}{cc}
O & A_{2}^{\prime} \\
O & A_{2}^{\prime \prime}
\end{array}\right), \quad U_{22}=\left(\begin{array}{cc}
U_{22}^{\prime} & O \\
O & U_{22}^{\prime \prime}
\end{array}\right)
$$

with respect to the decomposition $\mathcal{R}\left(A^{\pi}\right)=\mathcal{N}\left(U_{22}^{\pi}\right) \oplus \mathcal{R}\left(U_{22}^{\pi}\right)$, where $U_{22}^{\prime}$ is invertible, $A_{2}^{\prime \prime}$ and $U_{22}^{\prime \prime}$ are quasinilpotent with $A_{2}^{\prime \prime} U_{22}^{\prime \prime}=O$. By Lemma 2.6,

$$
\left|i\left(A_{2}^{\prime \prime}\right)-i\left(U_{22}^{\prime \prime}\right)\right|+1 \leq i\left(A_{2}^{\prime \prime}+U_{22}^{\prime \prime}\right) \leq i\left(A_{2}^{\prime \prime}\right)+i\left(U_{22}^{\prime \prime}\right)-1 .
$$

From $i\left(A_{2}^{\prime \prime}\right)+1 \geq i\left(A_{2}\right) \geq i\left(A_{2}^{\prime \prime}\right)$, we have

$$
\left|i\left(A_{2}\right)-i\left(U_{22}^{\prime \prime}\right)\right| \leq i\left(A_{2}^{\prime \prime}+U_{22}^{\prime \prime}\right) \leq i\left(A_{2}\right)+i\left(U_{22}^{\prime \prime}\right)-1 .
$$


From $i(B)=i\left(A_{2}+U_{22}\right)=i\left(A_{2}^{\prime \prime}+U_{22}^{\prime \prime}\right), i\left(A_{2}\right)=i(A)$ and $i\left(U_{22}^{\prime \prime}\right)=i\left(U_{22}\right)=i\left(A^{\pi} U\right)$, we have

$$
\left|i(A)-i\left(A^{\pi} U\right)\right| \leq i(B) \leq i(A)+i\left(A^{\pi} U\right)-1 .
$$

The following result is an extension of [2, Theorem 2.3].

Theorem 2.11. Let $A \in \mathcal{B}(X)$ be GD-invertible. Then there always exists a $\delta>0$ such that $A+U$ is $G D$-invertible whenever $U \in \mathcal{B}(X)$ is $A_{\delta}^{-1}$-compatible. In this case,

$$
(A+U)^{d}=\nu_{A}+\kappa_{A}-\nu_{A} U \kappa_{A}+\left[\sum_{n=0}^{\infty} \nu_{A}^{n+2} U A^{\pi}\left(A^{\pi}(A+U)\right)^{n}\right] \times\left[I-(A+U) \kappa_{A}\right],
$$

where $C_{A^{\pi} U}$ is invertible component in the core-quasinilpotent decomposition $A^{\pi} U=$ $C_{A^{\pi} U}+Q_{A^{\pi} U}$. In particular, if $-1 \notin \sigma\left[\left(A^{\pi} U\right)^{d} A^{\pi} U\left(I+\left(A^{\pi} U\right)^{d} A\right)\right]$, then

$$
\left|i\left(\left(A^{\pi} U\right)^{\pi} A\right)-i\left(A^{\pi} U\right)\right|+1 \leq i(A+U) \leq i(A)+i\left(A^{\pi} U\right)-1
$$

holds, provided that $i(A)>0$ and the difference $i\left[\left(A^{\pi} U\right)^{\pi} A\right]-i\left(A^{\pi} U\right)$ is defined.

Proof. Let $B=A+U$. Since $U$ is $A_{\delta}^{-1}$-compatible, $A^{\pi} U$ is GD-invertible and $A^{\pi} U$ has the core-quasinilpotent decomposition form $A^{\pi} U=C_{A^{\pi} U}+Q_{A^{\pi} U}$. If $A, U$ and $B$ have the representations as (2.2), then

$$
A^{\pi} U=O \oplus U_{22} \quad \text { and } \quad A_{2} U_{22}=U_{22} A_{2} .
$$

Let $P_{1}$ be the spectral idempotent of $U_{22}$ in the Banach algebra $\mathcal{B}\left(\mathcal{R}\left(A^{\pi}\right)\right)$ corresponding to $\{0\}$. Then $U_{22}$ has the form $U_{22}=U_{22}^{1} \oplus U_{22}^{2}$ with respect to the space decomposition $\mathcal{R}\left(A^{\pi}\right)=\mathcal{N}\left(P_{1}\right) \oplus \mathcal{R}\left(P_{1}\right)$, where $U_{22}^{1}$ is invertible with $C_{A^{\pi} U}=$ $O \oplus U_{22}^{1} \oplus O, U_{22}^{2}$ is quasinilpotent with $Q_{A^{\pi} U}=O \oplus O \oplus U_{22}^{2}$.

Since $A_{2}$ commutes with $P_{1}$, we have $A_{2}=A_{2}^{1} \oplus A_{2}^{2}$ with respect to the space decomposition $\mathcal{R}\left(A^{\pi}\right)=\mathcal{N}\left(P_{1}\right) \oplus \mathcal{R}\left(P_{1}\right)$, where $A_{2}^{i}(i=1,2)$ are quasinilpotent and $U_{22}^{i} A_{2}^{i}=A_{2}^{i} U_{22}^{i},(i=1,2)$. So $U_{22}^{2}+A_{2}^{2}$ is quasinilpotent and

$$
\left(A^{\pi} U\right)^{d} A^{\pi} U\left(I+\left(A^{\pi} U\right)^{d} A\right)=O \oplus\left(I+\left(U_{22}^{1}\right)^{-1} A_{2}^{1}\right) \oplus O .
$$

If $-1 \notin \sigma\left[\left(A^{\pi} U\right)^{d} A^{\pi} U\left(I+\left(A^{\pi} U\right)^{d} A\right)\right]$, then

$$
O \oplus\left(A_{2}+U_{22}\right)=O \oplus\left(U_{22}^{1}+A_{2}^{1}\right) \oplus\left(U_{22}^{2}+A_{2}^{2}\right)
$$

is GD-invertible. If $-1 \in \sigma\left[\left(A^{\pi} U\right)^{d} A^{\pi} U\left(I+\left(A^{\pi} U\right)^{d} A\right)\right]$, then by the definition of $A_{\delta}^{-1}$-compatible, -1 is the isolate point and so $I+\left(U_{22}^{1}\right)^{-1} A_{2}^{1}$ is GD-invertible. 
Note that $A_{2}^{1}, U_{22}^{1}$ and $\left(U_{22}^{1}\right)^{-1}$ commute pairwise, $U_{22}^{1}+A_{2}^{1}$ is GD-invertible and $\left(U_{22}^{1}+A_{2}^{1}\right)^{d}=\left(U_{22}^{1}\right)^{-1}\left(I+\left(U_{22}^{1}\right)^{-1} A_{2}^{1}\right)^{d}=\left(I+\left(U_{22}^{1}\right)^{-1} A_{2}^{1}\right)^{d}\left(U_{22}^{1}\right)^{-1}$. Hence

$$
O \oplus\left(A_{2}+U_{22}\right)=O \oplus\left(U_{22}^{1}+A_{2}^{1}\right) \oplus\left(U_{22}^{2}+A_{2}^{2}\right)
$$

is GD-invertible. By Lemma 2.4, we have

$$
\left(A^{\pi}(A+U)\right)^{d}=\left(O \oplus\left(A_{2}+U_{22}\right)\right)^{d}=C_{A^{\pi} U}^{d}\left(I+C_{A^{\pi} U}^{d} A\right)^{-1}=\kappa_{A} .
$$

Hence, by Lemma 2.2 and Lemma 2.3, there exists $\delta>0$, if $\|U\|<\delta$, then $A_{1}+U_{11}$ is invertible and $A_{2}+U_{22}$ is GD-invertible. It follows that $B=A+U$ is GD-invertible. By Lemma 2.7, we have

$$
B^{d}=\left(\begin{array}{cc}
\left(A_{1}+U_{11}\right)^{-1} & S \\
O & \left(A_{2}+U_{22}\right)^{d}
\end{array}\right),
$$

where

$\left.S=\left[\sum_{n=0}^{\infty}\left(A_{1}+U_{11}\right)^{-(n+2)} U_{12}\left(A_{2}+U_{22}\right)^{n}\right]\left(A_{2}+U_{22}\right)^{\pi}\right]-\left(A_{1}+U_{11}\right)^{-1} U_{12}\left(A_{2}+U_{22}\right)^{d}$.

Note that $\left(A_{1}+U_{11}\right)^{-1} \oplus O=\left(I+A^{d} U\right)^{-1} A^{d}=\nu_{A}$ and

$$
\left(\begin{array}{ll}
O & S \\
O & O
\end{array}\right)=\left[\sum_{n=0}^{\infty} \nu_{A}^{n+2} U A^{\pi}\left(A^{\pi}(A+U)\right)^{n}\right] \times\left[I-(A+U) \kappa_{A}\right]-\nu_{A} U \kappa_{A} .
$$

So we obtain

$$
B^{d}=\nu_{A}+\kappa_{A}-\nu_{A} U \kappa_{A}+\left[\sum_{n=0}^{\infty} \nu_{A}^{n+2} U A^{\pi}\left(A^{\pi}(A+U)\right)^{n}\right] \times\left[I-(A+U) \kappa_{A}\right] .
$$

From (2.2) we know $i(A)=i\left(A_{2}\right), i\left[\left(A^{\pi} U\right)^{\pi} A\right]=i\left(A_{2}^{2}\right)$ and $i(B)=i\left(A_{2}+U_{22}\right)$. Since $-1 \notin \sigma\left(\left(A^{\pi} U\right)^{D} A^{\pi} U\left(I+\left(A^{\pi} U\right)^{d} A\right)\right), i\left(A_{2}+U_{22}\right)=i\left(A_{2}^{2}+U_{22}^{2}\right)$ and $i\left(A_{2}\right) \geq$ $i\left(A_{2}^{2}\right)$. By Lemma 2.6, $\left|i\left(A_{2}^{2}\right)-i\left(U_{22}^{2}\right)\right|+1 \leq i\left(A_{2}^{2}+U_{22}^{2}\right) \leq i\left(A_{2}^{2}\right)+i\left(U_{22}^{2}\right)-1$. Hence

$$
\left|i\left(\left(A^{\pi} U\right)^{\pi} A\right)-i\left(A^{\pi} U\right)\right|+1 \leq i(B) \leq i(A)+i\left(A^{\pi} U\right)-1 .
$$

3. Special cases. Many interesting special cases of Theorem 2.10 and Theorem 2.11 were considered in $[2,23]$, Some of them are generalizations of the well-known results. Wei and Wang [23] considered a perturbation of the Drazin inverse of $A$ under the conditions on $U$ given by

$$
A A^{D} U A A^{D}=U \text { and }\left\|A^{D} U\right\|<1 .
$$


If $A$ and $U$ have the forms as (2.2), then condition (3.1) implies that $U_{i 2}=O(i=1,2)$. If $\delta$ is small enough, then the following result is a direct corollary of Theorem 2.10 and 2.11 .

Corollary 3.1. [23, Theorem 3.2] Suppose that $B=A+U$ with $U=A A^{d} U A A^{d}$ and $\left\|A^{d} U\right\|<1$ hold. Then

$$
\begin{gathered}
B^{d}-A^{d}=-B^{d} U A^{d}=-A^{d} U B^{d}, \\
B^{d}=\left(I+A^{d} U\right)^{-1} A^{d}=A^{d}\left(I+U A^{d}\right)^{-1}, \\
\frac{\left\|B^{d}-A^{d}\right\|}{\left\|A^{d}\right\|} \leq \frac{\left\|A^{d} U\right\|}{1-\left\|A^{d} U\right\|} .
\end{gathered}
$$

Castro González and Koliha [2] considered a perturbation of the Drazin inverse of $A$ under the $A$-compatible conditions on $U$ given by

$$
A^{\pi} U=U A^{\pi}, \quad \sigma\left(A^{\pi} U\right)=\{0\} \quad \text { and } \quad A A^{\pi} U=U A A^{\pi}
$$

and stated that $U$ is inverse- $A$-compatible (alternatively norm- $A$-compatible) if $U$ is $A$-compatible and satisfies $I+A^{d} U$ invertible (alternatively $\left\|A^{d} U\right\|<1$ ). Let $A$ and $U$ have the forms in (2.2). It is clear that if $U$ is inverse- $A$-compatible, then $U$ is $A_{\delta}^{-1}$-compatible with additional conditions $A_{1} U_{11}=U_{11} A_{1}, U_{12}=O, I+A_{1}^{-1} U_{11}$ invertible and $U_{22}$ quasinilpotent. These imply that $C_{A^{\pi} U}=O$ and $i\left(\left(A^{\pi} U\right)^{\pi} A\right)=$ $i(A)$. Then Theorem 2.3 in [2] is recovered as a special case of Theorem 2.11.

Corollary 3.2. [2, Theorem 2.3] Let $A \in \mathcal{B}(X)$ be GD-invertibIe and $U \in \mathcal{B}(X)$ be inverse- $A$-compatible. If $B=A+U$, then $B$ is $G D$-invertible, and

$$
\begin{aligned}
& \mathcal{N}\left(B^{\pi}\right)=\mathcal{N}\left(A^{\pi}\right) \text { and } \mathcal{R}\left(B^{\pi}\right)=\mathcal{R}\left(A^{\pi}\right), \\
& B^{d}=\left(I-A^{d} U\right)^{-1} A^{d}=A^{d}\left(I-U A^{d}\right)^{-1}
\end{aligned}
$$

and

$$
\left|i(A)-i\left(A^{\pi} U\right)\right|+1 \leq i(B) \leq i(A)+i\left(A^{\pi} U\right)-1
$$

holds, provided that $i(A)>0$ and the difference $i(A)-i\left(A^{\pi} U\right)$ is defined.

Let $A, U$ and $B$ have the forms in (2.2). In fact, the cases treated in [2] and [23] allow the perturbation of the invertible component of $A=A_{1} \oplus A_{2}$, and the quasinilpotent perturbation of the quasinilpotent component of $A=A_{1} \oplus A_{2}$. In Theorem 2.10 and Theorem 2.11, if $\delta$ is small enough, then $\left\|U_{11}\right\|<\delta$ and

$$
\left\|A^{d} U\right\|=\left\|A_{1}^{-1} U_{11} \oplus O\right\|=\left\|A_{1}^{-1}\right\| \delta<1 .
$$


So the following result is a direct corollary.

Corollary 3.3. [2, Corollary 2.5] Let $A \in \mathcal{B}(X)$ be GD-invertible and $B=A+U$ with $U \in \mathcal{B}(X)$ norm-A-compatible. Then

$$
\frac{\left\|B^{d}-A^{d}\right\|}{\left\|A^{d}\right\|} \leq \frac{\left\|A^{d} U\right\|}{1-\left\|A^{d} U\right\|}
$$

and

$$
\frac{\left\|A^{d}\right\|}{1+\left\|A^{d} U\right\|} \leq\left\|B^{d}\right\| \leq \frac{\left\|A^{d}\right\|}{1-\left\|A^{d} U\right\|}
$$

4. Concluding remarks. In this paper, we investigate the perturbation of the generalized Drazin invertible operators and derive explicit generalized Drazin inverse expressions for the perturbations under certain restrictions on the perturbing operators. It is natural to ask if we can extend our results to the $\mathrm{W}$-weighted Drazin inverse $[6,10,21]$, which will be the future research topic.

Acknowledgments. The authors would like to thank referees for their very detailed comments which greatly improve the presentation of our paper.

\section{REFERENCES}

[1] S. L. Campbell and C. D. Meyer. Continuity properties of the Drazin pseudoinverse. Linear Algebra Appl, 10:77-83, 1975.

[2] N. Castro González and J. Koliha. Perturbation of the Drazin inverse for closed linear operators. Integr. Equ. Oper. Theory, 36:92-106, 2000.

[3] N. Castro González, J. Koliha, and V. Rakočević. Continuity and general perturbation of the Drazin inverse for closed linear operators. Abstr. Appl. Anal., 7:335-347, 2002.

[4] N. Castro González, J. Koliha, and Y. Wei. Error bounds for perturbation of the Drazin inverse of closed operators with equal spectral projections. Appl. Anal., 81:915-928, 2002.

[5] N. Castro González and J. Koliha. New additive results for the $g$-Drazin inverse. Proc. Roy. Soc. Edinburgh Sect. A, 134:1085-1097, 2004.

[6] N. Castro-González and J. Vélez-Cerrada. The weighted Drazin inverse of perturbed matrices with related support idempotents. Appl. Math. Comput., 187:756-764, 2007.

[7] N. Castro González and J.Y. Vélez-Cerrada. On the perturbation of the group generalized inverse for a class of bounded operators in Banach spaces. J. Math. Anal. Appl., 341:1213-1223, 2008.

[8] D. Cvetković-Ilić, D. Djordjević, and Y. Wei. Additive results for the generalized Drazin inverse in a Banach algebra. Linear Algebra Appl., 418:53-61, 2006.

[9] D. Cvetković-Ilić and Y. Wei. Representations for the Drazin inverse of bounded operators on Banach space. Electronic Journal of Linear Algebra, 18:613-627, 2009.

[10] A. Dajić and J. Koliha. The weighted $g$-Drazin inverse for operators. J. Aust. Math. Soc., 82:163-181, 2007. 
[11] C. Y. Deng and H. K. Du. The reduced minimum modulus of Drazin inverses of linear operators on Hilbert spaces, Proc. Amer. Math. Soc., 134:3309-3317, 2006.

[12] D. S. Djordjević and Y. Wei. Additive results for the generalized Drazin inverse. J. Austral Math. Soc., 73:115-125, 2002.

[13] D. S. Djordjević and P. S. Stanimirović. On the generalized Drazin inverse and generalized resolvent. Czechoslovak Math. J., 51(126):617-634, 2001.

[14] H. K. Du and C. Y. Deng. The representation and characterization of Drazin inverses of operators on a Hilbert space. Linear Algebra Appl., 407:117-124, 2005.

[15] J. K. Han, H. Y. Lee, and W. Y. Lee. Invertible completion of $2 \times 2$ upper triangular operator matrices. Proc. Amer. Math. Soc., 128:119-123, 2000.

[16] R. Hartwig, X. Li, and Y. Wei. Reprsentations for the Drazin inverse of $2 \times 2$ block matrix. SIAM J. Matrix Anal. Appl., 27:757-771, 2006.

[17] J. Koliha and V. Rakočević. Continuity of the Drazin inverse II. Studia Mathematica, 131(2):167-177, 1998.

[18] J. J. Koliha. A generalized Drazin inverse. Glasgow Math. J., 38:367-381, 1996.

[19] X. Li and Y. Wei. An expression of the Drazin inverse of a perturbed matrix. Appl. Math. Comput., 153:187-198, 2004.

[20] V. Rakočević and J. Koliha. Drazin invertible operators and commuting Riesz perturbations. Acta Sci. Math. (Szeged), 68:291-301, 2002.

[21] V. Rakočević and Y. Wei. A weighted Drazin inverse and applications. Linear Algebra Appl., 350:25-39, 2002.

[22] V. Rakočević and Y. Wei. The perturbation theory for the Drazin inverse and its applications II. J. Aust. Math. Soc., 70:189-197, 2001.

[23] Y. Wei and G. Wang. The perturbation theory for the Drazin inverse and its applications. Linear Algebra Appl., 258:179-186, 1997.

[24] Y. Wei. The Drazin inverse of updating of a square matrix with application to perturbation formula. Appl. Math. Comput., 108:77-83, 2000.

[25] Y. Wei and H. Wu. The perturbation of the Drazin inverse and oblique projection. Appl. Math. Lett., 13:77-83, 2000

[26] Y. Wei and H. Wu. Challenging problems on the perturbation of Drazin inverse. Ann. Oper. Res., 103:371-378, 2001.

[27] Y. Wei. Perturbation bound of the Drazin inverse. Appl. Math. Comput., 125:231-244, 2002.

[28] Y. Wei and X. Li. An improvement on perturbation bounds for the Drazin inverse. Numer. Linear Algebra Appl., 10:563-575, 2003.

[29] Y. Wei, X. Li, and F. Bu. A perturbation bound of the Drazin inverse of a matrix by separation of simple invariant subspaces. SIAM J. Matrix Anal. Appl., 27:72-81, 2005.

[30] Q. Xu, C. Song, and Y. Wei. The stable perturbation of the Drazin inverse of the square matrices. SIAM J. Matrix Anal. Appl., 31:1507-1520, 2010. 